\title{
Segregation scheme of indium in AlGaInAs nanowire shells
}

\author{
Luca Francaviglia, ${ }^{1}$ Gözde Tütüncüoglu, ${ }^{1}$ Sara Martí-Sánchez, ${ }^{2}$ Enrico Di Russo, ${ }^{3}$ Simon Escobar Steinvall, ${ }^{1}$ \\ Jaime Segura Ruiz, ${ }^{4}$ Heidi Potts, ${ }^{1}$ Martin Friedl, ${ }^{1}$ Lorenzo Rigutti, ${ }^{3}$ Jordi Arbiol, ${ }^{2,5}$ and Anna Fontcuberta i Morral ${ }^{1,6, *}$ \\ ${ }^{1}$ Laboratoire des Matériaux Semiconducteurs, Institut des Matériaux, École Polytechnique Fédérale de Lausanne, \\ 1015 Lausanne, Switzerland \\ ${ }^{2}$ Catalan Institute of Nanoscience and Nanotechnology (ICN2), CSIC and BIST, Campus UAB, Bellaterra, 08193 Barcelona, Catalonia, Spain \\ ${ }^{3}$ Groupe de Physique des Matériaux, Université de Rouen, Saint-Étienne-du-Rouvray, 76801, France \\ ${ }^{4}$ European Synchrotron Radiation Facility, Grenoble, 38043, France \\ ${ }^{5}$ ICREA, Pg. Lluís Companys 23, 08010 Barcelona, Catalonia, Spain \\ ${ }^{6}$ Institute of Physics, École Polytechnique Fédérale de Lausanne, CH-1015, Switzerland
}

(Received 26 November 2018; published 13 February 2019)

\begin{abstract}
Quaternary alloys enable the independent optimization of different semiconductor properties, such as the separate tuning of the band gap and the lattice constant. Nanowire core-shell structures should allow a larger range of compositional tuning as strain can be accommodated in a more effective manner than in thin films. Still, the faceted structure of the nanowire may lead to local segregation effects. Here, we explore the incorporation of indium in $\mathrm{AlGaAs}$ shells up to 25\%. In particular, we show the effect of In incorporation on the energy shift of the AlGaInAs single-photon emitters present in the shell. We observe a redshift up to $300 \mathrm{meV}$ as a function of the group-III site fraction of In. We correlate the shift with segregation at the nanoscale. We find evidence of the segregation of the group-III elements at different positions in the nanowire, not observed before. We propose a model that takes into account the strain distribution in the nanowire shell and the adatom diffusion on the nanowire facets to explain the observations. This work provides novel insights on the segregation phenomena necessary to engineer the composition of multidinary alloys.
\end{abstract}

DOI: 10.1103/PhysRevMaterials.3.023001

Introduction. Quaternary alloys enable to independently tune the semiconductor lattice constant and band gap by careful composition engineering. However, the controlled deposition of quaternary alloys with randomly distributed composition can be challenging. Miscibility gaps and diffusion may induce the segregation of the different species that compose the alloy, and limit the possible compositions [1,2]. Moreover, strain may build up when quaternary semiconductors epitaxially grow on substrates of different lattice constant. To avoid plastic relaxation $[3,4]$, the theoretical range of available compositions is significantly narrowed in real applications. Due to their reduced diameter, semiconductor nanowires (NWs) provide a suitable growth platform to minimize plastic relaxation, [5-10] permitting lattice-mismatched material combinations not achievable by planar schemes [11-14].

In this work, we explore the range in which In can be incorporated in AlGaAs shells of core-shell GaAs-AlGaAs NWs. The goal is to understand how much the emission energy of single-photon emitters, spontaneously forming in the shell, can be redshifted [15-17]. In particular, we want to dissociate the band-gap engineering from additional segregation effects that may occur due to the presence of In. Consequently, we characterize the optical emission and correlate it with the incorporation of In using several techniques at different length scales. We find that the group-III elements in the shell segre-

\footnotetext{
*anna.fontcuberta-morral@epfl.ch
}

gate to different positions and generate regions of different band gaps. In particular, we find consistent evidence of the In segregation in novel wedge-shaped In-rich features. We correlate these observations with the threefold polarity of the NW cross section and the migration of the adatoms on the NW sidewalls. In addition to the role of the crystal directions of the NW facets [18-20], we consider strain to cause the peculiar In segregation; the presence and distribution of strain are further analyzed by finite-element simulations as well as Raman and photoluminescence (PL) spectroscopy.

The material system: core-shell NWs. The GaAs$\mathrm{AlGa}(\mathrm{In}) \mathrm{As}$ core-shell NWs are grown in a high-mobility molecular-beam-epitaxy system (MBE, DCA P600). We use $\mathrm{Si}(111)$ substrates and self-catalyzed growth $[23,24]$ at a substrate temperature of $640{ }^{\circ} \mathrm{C}$. When the NWs are about $10-\mu \mathrm{m}$ long, we stop the axial growth by interrupting the Ga supply and consuming the catalyst. We then lower the substrate temperature to $460{ }^{\circ} \mathrm{C}$ to promote radial growth of an $\mathrm{AlGa}(\mathrm{In}) \mathrm{As}$ shell on the NW sidewalls [25,26]. We start with an $\mathrm{Al}_{33} \mathrm{Ga}_{67} \mathrm{As}$ shell and then incorporate In without modifying the $\mathrm{Ga}$ or $\mathrm{Al}$ rates. For this, we varied the In partial pressure from $4.5 \times 10^{-9}$ to $2.5 \times 10^{-7}$ Torr, while the $\mathrm{Al}$ and $\mathrm{Ga}$ pressures were kept constant. Unless stated, the samples are identified by the group-III site fraction of In measured through various techniques and expressed as percentage in the notation $\mathrm{Al}_{x} \mathrm{Ga}_{y} \mathrm{In}_{1-x-y} \mathrm{As}(\mathrm{Al}, \mathrm{Ga}$, and In sum to $100 \%): 0 \%, 1 \%, 2 \%, 3 \%, 4 \%, 15 \%$, and $25 \%$. An outer GaAs shell of $5 \mathrm{~nm}$ prevents the oxidation of the inner $\mathrm{AlGa}(\mathrm{In}) \mathrm{As}$. 
Previous reports show that the composition of an $\mathrm{AlGaAs}$ shell deposited around a GaAs NW exhibits nanoscale fluctutations $[15,16,27-30]$. This phenomenon depends on the different surface mobility of $\mathrm{Ga}$ and $\mathrm{Al}$ during growth and the presence of surface-potential gradients $(\mu)$ on the NW sidewalls. The formation of Al-rich planes at the ridge between two NW facets was reported [19]. Furthermore, Garich clusters can form quantum-dots (QDs) that behave as single-photon emitters in the NW shell $[15,16,29]$. A sketch of a core-shell GaAs-AlGaAs NW and its cross section is presented in Fig. 1(a), illustrating the distribution of Al-rich planes and QDs made from Ga-rich nanoclusters.

Light-emission properties. First we address the optical functionality of the core-shell NWs and embedded QDs. We used a single-frequency optically pumped semiconductor laser, with a wavelength of $532 \mathrm{~nm}$, focused in a spot of less than $1 \mu \mathrm{m}$ in diameter $\left(100 \mathrm{~W} / \mathrm{cm}^{2}\right)$ to measure $\mathrm{NW}$ samples at $12 \mathrm{~K}$ using a helium cryostat. The PL signal is collected into a spectrometer and dispersed by a $300 \mathrm{l} / \mathrm{mm}$ grating onto a Peltier-cooled CCD. The QDs in the AlGaAs shell emit bright and narrow PL lines (linewidth below $100 \mu \mathrm{eV}$ ) [15] between 1.7 and $1.9 \mathrm{eV}$ [27]. The green spectrum at the bottom of Fig. 1(b) illustrates the PL emission of these structures; the sharp peaks are attributed to the presence of QD single-photon emitters [15,31]. Figure 1(b) also contains PL spectra of the shell-QD emission for increasing In fraction, revealing several sharp peaks at different energies. The emission-energy range redshifts with increasing In fraction in the shell. One can also qualitatively determine that the QD emission linewidth broadens in samples of higher In fraction. The sample with the highest In fraction showed a decreased PL intensity, compensated for by increasing the laser power [see Fig. 1(b)].

For a statistical analysis on large ensembles of NWs and QDs, Fig. 1(c) shows the median emission energy of the QDs as a function of the measured In pressure in the MBE chamber (bottom axis) or as a function of the In fraction (top axis) measured in the shell by $\mathrm{x}$-ray fluorescence (XRF) combined with atom probe tomography (APT) for $1 \%$ to $4 \%$ of In and scanning transmission electron microscopy energy-dispersive $\mathrm{x}$-ray spectroscopy (STEM EDX) for $15 \%$ and $25 \%$ In, see Ref. [32]. We measured $25 \mathrm{NWs}$ from each sample. The QD emission lines were identified by an automatic routine [27]. The dashed-dotted line indicates the expected shell band gap as a function of shell composition [21]. The dashed line corresponds to the GaAs band gap. Both lines are corrected for the simulated strain [22] that arises from the core-shell lattice mismatch, as further discussed in the manuscript.

The inset in Fig. 1(c) shows a Hanbury-Brown-Twiss autocorrelation measurement $\left[\mathrm{g}^{2}(\tau)\right]$ of the exciton line of a QD from a sample with 3\% In. The power-dependent PL showing the exciton nature of the emission line is reported in the supporting information. The sample is measured at $4.2 \mathrm{~K}$ in an Attodry 700 closed-cycle cryostat and is excited in continuous wave by a $\mathrm{HeNe}$ laser at $632.8 \mathrm{~nm}$ through an objective with $\mathrm{NA}=0.81$, which also collects the QD signal from the cryostat. A $12001 / \mathrm{mm}$ grating is used to select the QD line of interest that is sent to a 50:50 beam splitter. The two paths of the beam splitter are coupled to single-mode optical fibers that send the signal to two single-photon avalanche diodes, one for each path. The dip in the autocorrelation function in
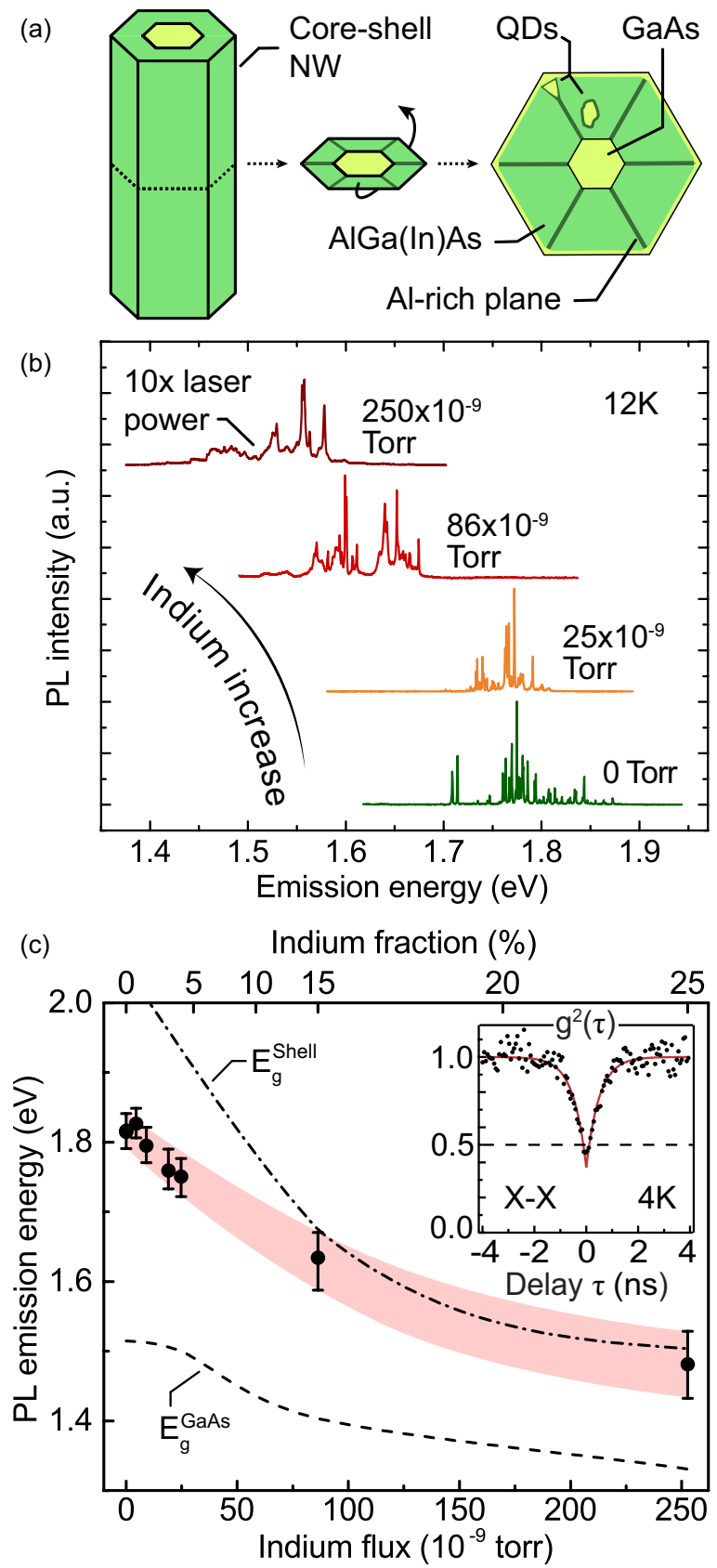

FIG. 1. (a) Sketch of a core-shell NW and shell QDs. (b) MicroPL spectra of shell QDs grown at increasing In pressures. (c) Ensemble medians of the PL QD emission energy vs the In MBE pressure (bottom abscissa) or shell fraction (top abscissa) given by APT, XRF, STEM EDX, and growth-rate calibrations. The distributions first and third quartiles act as error bars, highlighted, as a guide to the eye, by the shaded area. The dashed and the dashed-dotted lines are the strained core and shell band gaps, respectively [21,22]. (Inset) Single-photon $\mathrm{g}^{2}$ of a QD exciton (3\% In).

the inset of Fig. 1(c) is below 0.5, which is the signature of the single-photon emission. Several factors increase the count at zero delay, including background counts from QD lines spectrally close to the chosen one.

The effect of the shell-composition engineering on the QD emission energy is significant. From Fig. 1(c), the median QD 
emission redshift is visible as a function of the increasing In incorporation, by up to about $300 \mathrm{meV}$. In a randomdistribution alloy, the addition of In to AlGaAs lowers the shell band gap and the QD emission energy. Since lowerband gap nanoscale clusters in the $\mathrm{AlGa}(\mathrm{In}) \mathrm{As}$ matrix form the studied QDs, their emission is always expected to be redshifted with respect to the band gap of the host shell matrix [dashed-dotted line in Fig. 1(c)]. In Fig. 1(c), we observe that the energy difference between the QD emission and the shell band gap is gradually reduced for higher In fractions. This calls for a different segregation effect at low and high In fractions, whose mechanisms and details may be investigated in a further study.

Compositional analysis at the nanoscale. In NWs the composition can vary significantly at the nanometer scale due to their faceted nature. The hexagonal cross-section of a GaAs NW exhibits six $\{110\}$ facets. The ridges between two of these facets are $\{112\}$ nanofacets with alternating A- and B-polar (Ga- and As-terminated) surfaces [18,19,30]. $\{110\}$ and $\{112\}$ facets have different and species-dependent sticking coefficients [30]. In addition, the increased surface curvature at the ridges increases the local surface energy and affects the adatom mobility. Here, we provide compositional measurements with spatial resolution down to the nanometer scale: APT and STEM-based electron energy loss spectroscopy (EELS) and EDX.

We performed laser-assisted APT measurements on the sample with $2 \% \mathrm{In}$. The specimen is cooled to $80 \mathrm{~K}$ and irradiated with UV laser light (343-nm wavelength) in 2-nJ pulses; the detection rate is 0.0025 events/pulse. The evaporated NW volume is a cylinder with diameter of $64 \mathrm{~nm}$ and length of $90 \mathrm{~nm}$; in Figs. 2(a)-2(c), it is shown as a 2D projection on a plane perpendicular to the NW growth axis. Figures 2(a)-2(c) show, respectively, the In, $\mathrm{Al}$, and $\mathrm{Ga}$ fractions. It is possible to distinguish the GaAs core by the absence of In and Al. In the shell, radial segregation of $\mathrm{Al}$ along the ridges between two facets of the hexagonal NW core is visible as the three Al-rich stripes in the reconstructed NW volume, in agreement with previous works $[15,16,18,19,29,30]$. In addition to the Al-rich planes, APT reveals that the shell distribution of the other group-III atoms is not perfectly random throughout the NW shell. A slightly higher In fraction (up to $3 \%$ ) is visible in proximity of two of the Al rich planes indicated as 1 and 3 in Fig. 2(a). Farther from these positions, the average In fraction decreases to about $1.6 \%$. In Figs. 2(b) and 2(c), we can also observe that the shell in proximity of the Al-rich planes is slightly depleted in $\mathrm{Al}$ and enriched in Ga. At shorter length scales, APT-based distributions [16] of the distances between first-neighbor atoms in the shell do not evidence short-range reordering of the quaternary alloy (Fig. S13 in Ref. [32]).

To study the segregation of $\mathrm{Al}, \mathrm{Ga}$, and In in more extended regions, we studied the NW cross-sections by high angular annular dark field (HAADF) STEM and EELS in an aberrationcorrected (AC) TEM microscope (FEI Titan) operated at 300 $\mathrm{keV}$. We used the sample with $15 \%$ In. We prepared NW cross sections to directly map the shell composition: the NWs were spread on a Si substrate and the cross sections were FIB-cut perpendicularly to the NW growth direction $(\langle 111\rangle)$. The cross-section HAADF micrographs are acquired along the $\langle 111\rangle$ zone axis, with the NW sidewalls corresponding to the $\{110\}$ family (Fig. S4 in Ref. [32]). Figure 2(d) reports the HAADF micrograph of one NW cross section. The HAADF contrast depends on the sample thickness and composition: the higher the atomic number $(Z)$ of the species in the sample, the brighter the HAADF signal. Through high-precision FIB cut, the NW cross sections have negligible thickness variations: the contrast in Fig. 2(d) depends on the average $Z$ at different positions across the sample. In this figure, the hexagonal shape of the NW cross section is clearly visible and the core is distinguishable from the shell. The dark stripes that form in the shell along the ridges between two NW facets $(\langle 112\rangle$ crystalline directions) are due to local enrichment with a light element, such as Al, as already observed by APT and in agreement with the literature $[15,18,20]$. It is possible to observe that the thickness of the Al-rich planes follows a threefold symmetry as observed by Zheng et al. [18]. We obtain a thickness of 3 and $1.7 \mathrm{~nm}$ for the thick and thin planes, respectively (more details in Fig. S4 in Ref. [32]). Similarly, the HAADF contrast shows that the thick planes are richer in $\mathrm{Al}$ than the thin ones. Comparing our observations with the literature [18], we assign the thin planes to the A polarity and the thick ones to the B polarity. Accordingly, we labeled the two polarities in Fig. 2(d) as A (orange) and $\mathrm{B}$ (blue). We highlight that, without the alternation of the $\mathrm{A}$ and $B$ polarity of the $\{112\}$ nanofacets, all the corners of the hexagonal NW cross-section would be equivalent.

With the exception of the Al-rich planes, the HAADF micrograph in Fig. 2(d) shows little compositional contrast in the shell. By carefully inspecting Fig. 2(d) and Fig. S4 in Ref. [32], a slightly brighter contrast is visible in proximity of the Al-rich planes: it corresponds to a local increase in the fraction of elements with higher $Z$, such as Ga and In. To clarify this observation, in Figs. 2(e)-2(h), we report the EELS maps of the upper half of the NW cross-section shown in Fig. 2(d): the selected region, indicated by the dashed rectangle in Fig. 2(d), includes an A-polar and two B-polar ridges as well as part of the $\{110\} \mathrm{NW}$ sidewalls. The Ga, In, Al, and As distributions are shown in Figs. 2(e)-2(h), respectively. Principal component analysis (PCA) was used to enhance the signal-to-noise ratio in these maps $[33,34]$. The NW core is clearly distinguished from the shell by the absence of $\mathrm{Al}$ and In and a thin Ga-rich layer, corresponding to the GaAs capping, surrounds the shell [Fig. 2(e)]. As is randomly distributed throughout [Fig. 2(f)], as opposed to the distributions of the group-III elements: the presence of Al-rich planes along the hexagon ridges is confirmed [Fig. 2(g)], together with the $\mathrm{Ga}$ and In depletion at the same positions [Figs. 2(e) and 2(f)]. Reports on GaAs-InGaAs and GaAsInAlAs core-shell NWs [35] also show Ga and In depletion along the $\langle 112\rangle$ direction. The brightness and thickness of the Al-rich planes agree with the threefold polarity-driven segregation previously discussed.

In agreement with the localized In enrichment shown by APT [Fig. 2(a)], STEM EELS shows in Fig. 2(f) In-rich features surrounding the Al-rich planes at the $\langle 112\rangle \mathrm{B}$ directions: interestingly, moving from the core to the outer shell, the In-rich regions become broader with a wedge-like shape on each side of the Al-rich B-polar planes. Ga has a more random distribution in the shell, although a slight increase is visible around the A-polar $\{112\}$ nanofacet in Fig. 2(e). This Ga-rich 
(a)
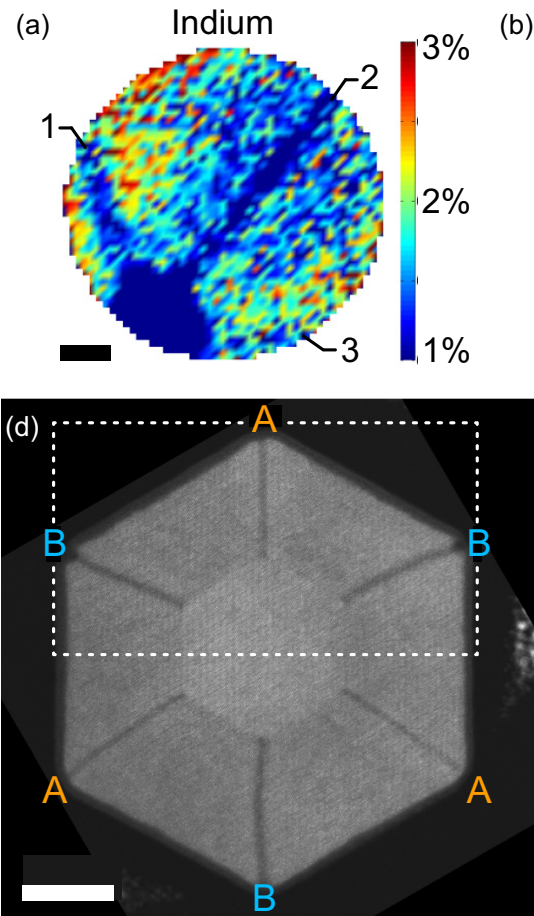

(i)

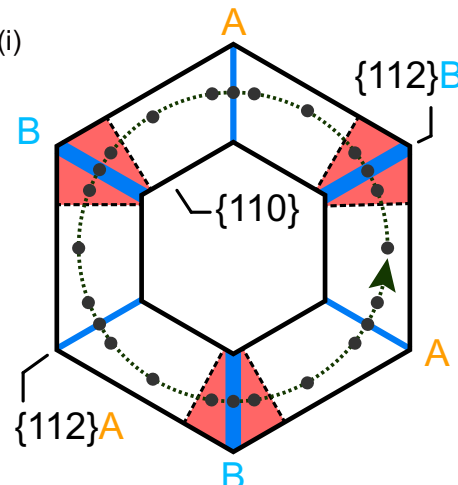

(b)
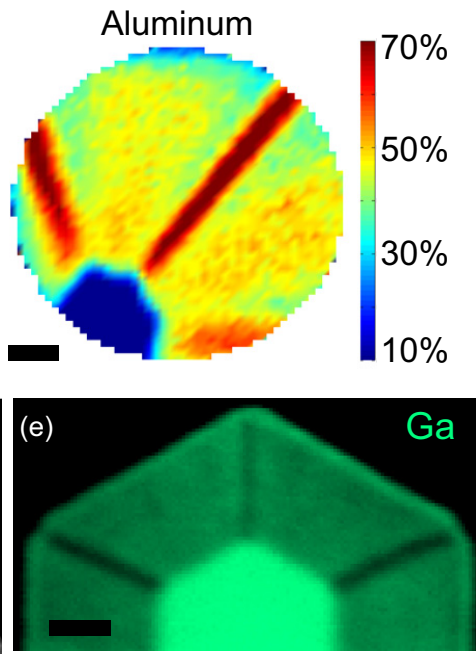

(g)

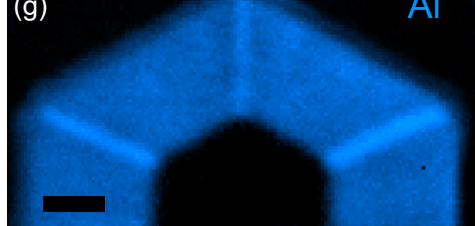

(c)
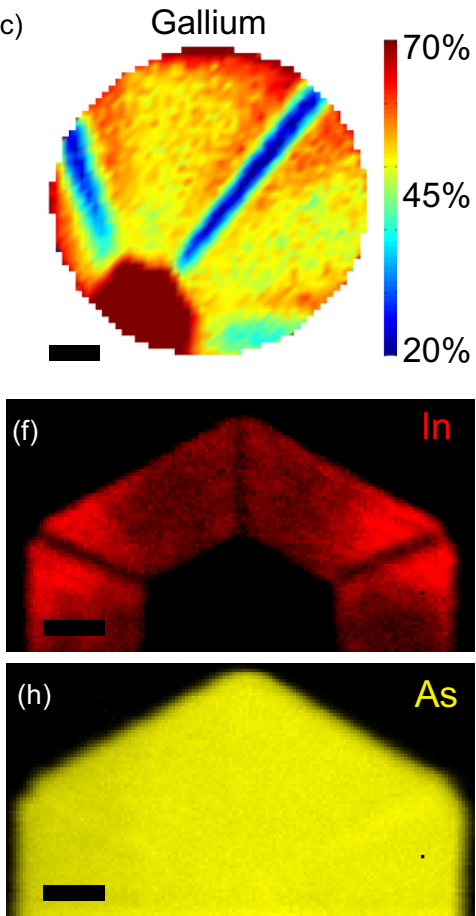

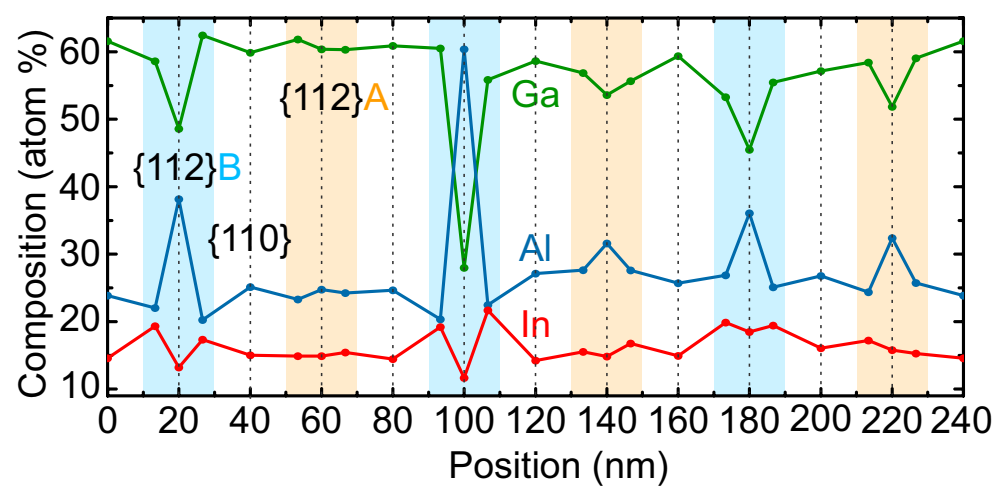

FIG. 2. [(a)-(c)] APT of In, Al, and Ga fractions (2\% In). (d) HAADF STEM micrograph of a NW cross section (15\% In) with A and B polarity indicated. [(e)-(h)] EELS Ga, In, Al, and As maps of the dashed rectangle in (d). (i) Left: sketch of shell segregation. Right: shell Ga (green), Al (blue), and In (red) atomic group-III percentages vs position along the circumference in the sketch. Scale bars: (a)-(c) and (e)-(h) $10 \mathrm{~nm}$ and (d) $20 \mathrm{~nm}$.

feature is not as pronounced as the In-rich ones surrounding the B-polar planes; it also has a relatively constant thickness around the A-polar planes, while the In-rich features develop an unusual wedge-shaped profile. One may also observe that, in proximity of the Al-rich planes aligned in the $\langle 112\rangle$ directions of both polarities, the $\mathrm{Al}$ fraction slightly decreases [Fig. 2(g)]. Although the contrast is not sharp, this would agree with the local increase in the $\mathrm{Ga}$ and In fractions at the same positions. EELS maps on whole NW cross sections confirm the threefold symmetry of the shell segregation and the In enrichment only around the three $\langle 112\rangle \mathrm{B}$ directions (see the supporting information). On the left of Fig. 2(i), a scheme summarizes the main compositional segregations observed in the NW cross sections [the scheme is aligned with Figs. 2(d)2(h)]: the red-shaded areas indicate the wedge-shaped Inrich segregation and blue stripes indicate the alternatively thicker and thinner Al-rich planes. The polarity of the $\{112\}$ nanofacets is labeled.
We acquired STEM-EDX maps of NW cross sections from the same samples as the one used for EELS. We use a FEI Tecnai Osiris TEM operated in STEM mode at $200 \mathrm{kV}$ with a probe current exceeding $1 \mathrm{nA}$. The $\mathrm{x}$-ray signal is collected by four silicon drift detectors under a solid angle of $0.9 \mathrm{srad}$. The NW cross sections are prepared by embedding the as-grown sample into epoxy. After hardening, the epoxy with embedded NWs is detached from the growth substrate and is mounted into an ultramicrotome and cut into 80-nm-thick slices transferred on a TEM grid. The EDX maps (Fig. S3 in Ref. [32]) for the $\mathrm{Ga}, \mathrm{Al}$, and In distributions in the core-shell cross sections confirm the presence of the same features observed in the EELS maps. Our observations are particularly robust: three independent techniques (APT, EELS, and EDX) confirm the formation of novel threefold wedge-shaped In-rich segregates in NWs with different average In fractions; together with the clear symmetry of these features, the agreement of the three techniques excludes artifacts due to sample preparation. 
We now turn to a quantitative analysis of the distribution of the different species in the NW cross section: the EDXbased quantification is more accurate than the one based on EELS [36]. Figure 2(i) shows on the right a plot of the Ga (green curve), Al (blue curve), and In (red curve) atomic percentage extracted from a STEM EDX map as a function of the position along the dashed circumference shown in the sketch on the left (i.e., a circular linescan of $240 \mathrm{~nm}$ in length). The data points at 0 and $240 \mathrm{~nm}$ correspond to the same position on the circumference (arrow in the sketch). The shaded areas in the plot are colored in orange and blue with the same coding as in Fig. 2(d) to distinguish the A- and B polarity of the $\{112\}$ nanofacets, respectively. The data points acquired in the middle of the flat $\{110\}$ facets have a white background.

We focus on the most prominent fluctuation around the $\langle 112\rangle \mathrm{B}$ facet at position $100 \mathrm{~nm}$. Moving from the $\{110\}$ plane to the $\{112\} \mathrm{B}$ nanofacet, the $\mathrm{Al}$ fraction first decreases (from $25 \%$ to $20 \%$ ), then rises to $60 \%$ at the corner. In smaller proportions, the In distribution is opposed to the Al trend: for the same positions, In first increases (from 14\% to 19\%) and then decreases to $12 \%$ at the corner. $\mathrm{Ga}$ is almost constant at $61 \%$ and $60 \%$ and then decreases to $28 \%$ at the corner. Very similar trends in $\mathrm{Al} / \mathrm{Ga} / \mathrm{In}$ fractions are reproduced at the six corners of the hexagonal NW cross section and are modulated according to a threefold symmetry: the compositional variations are consistently more pronounced in proximity of the B-polar facets.

Strain analysis and growth model. We turn now to the understanding of the element distribution in the shell by taking strain into account. Figure 3(a) shows the PL spectra of the GaAs core at $12 \mathrm{~K}$ for core-shell NWs of increasing In fraction. The band-edge GaAs PL redshifts from $1.51 \mathrm{eV}$ down to 1.37 and $1.29 \mathrm{eV}$ for shells containing $15 \%$ and $25 \% \mathrm{In}$, respectively, which we attribute to the tensile strain imposed by the shell. Tensile strain in the core is also confirmed by Raman spectroscopy [32].

To gain insight in the strain distribution imposed by the lattice-mismatched core-shell $\mathrm{NW}$, we simulated the strain given the lattice mismatch, dimensions and geometry of the structure. We use the methodology of Boxberg et al. [37], adapted to hexagonal GaAs-AlGaInAs NWs at low temperature [21] in the software NEXTNANO [38,39]. The strain magnitude and distributions in the core and shell are calculated by minimizing the elastic energy due to the lattice mismatch between the two. Figure 3(b) shows a map of the hydrostatic strain $\epsilon_{\text {hydro }}$ in a NW with $25 \%$ In in the shell. $\epsilon_{\text {hydro }}$ is dominated by the principal component along the NW longitudinal axis, as reported elsewhere [40]. Shear strain components are one order of magnitude smaller than $\epsilon_{\text {hydro }}$, in
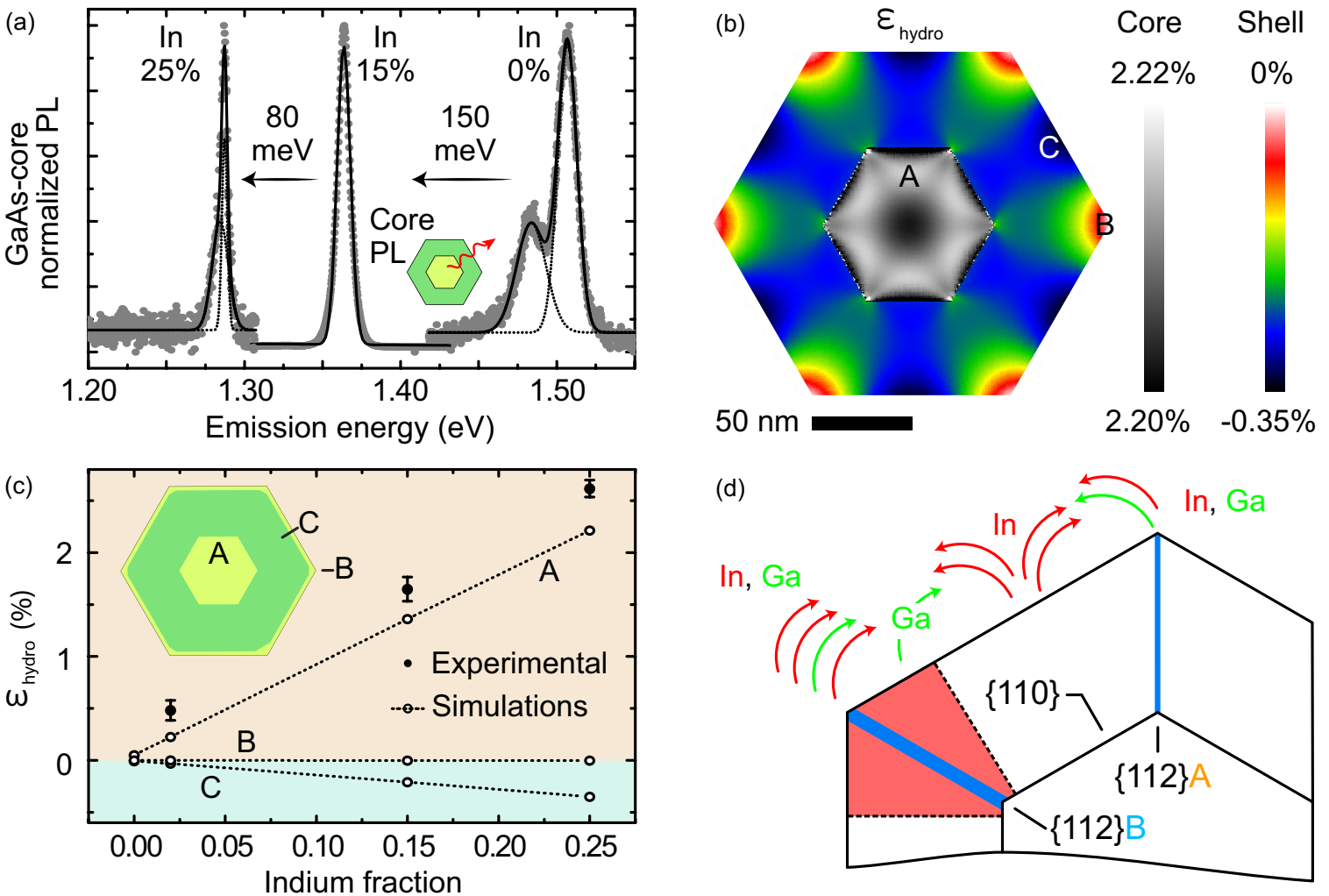

FIG. 3. (a) GaAs PL of NWs with In shell fraction of $0 \%, 15 \%$, and $25 \%$, acquired at $12 \mathrm{~K}$ with excitation laser of wavelength of $532 \mathrm{~nm}$ and power density of $100 \mathrm{~W} / \mathrm{cm}^{2}$. (b) Hydrostatic strain simulation in core-shell NWs (25\% In) as in Ref. [37]. (c) Simulated and average experimental ( $1 \sigma$ error bar) hydrostatic strain vs In fraction. (d) Sketch of the Ga and In diffusion on the NW sidewalls. 
agreement with previous reports [40]. As expected from the core-shell lattice mismatch, $\epsilon_{\text {hydro }}$ is on average negative in the shell (compressive) and positive in the core (tensile). We find about 2.2\% of tensile strain [position "A" in Fig. 3(b)], softly modulated following a sixfold symmetry. The shell is subject to a maximum compression of $-0.35 \%$ in the middle of the NW facet [position " $C$ " of Fig. 3(b)]. The shell strain intensity is modulated by a sixfold symmetry in a more pronounced way. The outer corners are relaxed [minimum strain of $0 \%$ at position "B" in Fig. 3(b)]. Importantly, the regions of smaller strain in the shell expand around the NW sidewall ridges, becoming larger the farther they are from the core. The experimental (PL) [41] and simulated strain values are plotted in Fig. 3(c): the simulated compressive strain in the shell (position C) and the experimental and simulated tensile strain in the core increase linearly with the In fraction. The theoretical and experimental values agree within $15 \%$.

A growth model that explains the nonrandom distribution of In and $\mathrm{Al}$ in the shell is presented. The expected behavior of $\mathrm{Ga}$ and In adatoms at the NW facets is sketched in Fig. 3(d). We start by addressing the $\mathrm{Al}$ distribution. $\mathrm{Al}$ tends to incorporate more efficiently at the vertices of the hexagonal cross section (\{112\} nanofacets) $[15,18,19]$. This is the consequence of a larger sticking coefficient on those facets $[42,43]$. For a similar reason, it has been shown that the incorporation is higher on B- than A-polar facets [18]. This results in a threefold symmetry of the $\mathrm{Al}$ distribution.

We now turn to the incorporation of In. According to our simulations, there is a compressive strain in the middle of the facets (position $\mathrm{C}$ in the drawings). Since AlGaInAs exhibits a larger lattice constant than AlGaAs, we expect In to be favorably incorporated in the relaxed corners. In adatoms exhibit high mobilities, allowing diffusion to occur at the scale of the NW facets. Strain relaxation should support an In flux towards the more relaxed $\{112\}$ corner nanofacets, while the $\{110\}$ facets exhibit a significantly lower In fraction in the center [arrows leaving this position in Fig. 3(d)]. Qualitatively, there is good match between the wedge-shaped In segregation and the simulated strain distributions that show increasing strain relaxation towards the outer NW shell. This correlation suggests that strain has an important role in shaping the In segregation. It is also known that moving steps in step-flow or 2D-island growth may drive the atom segregation [44]. Here, it is difficult to assess how atomic steps affect the In diffusion without performing in-situ experiments. However, the steps may enhance, but definitely do not significantly prevent, the strain-driven segregation of In at the NW ridges. In highly lattice-mismatched GaAs-InGaAs core-shell NWs [5], the coherent shell deposition on the $\{110\}$ sidewalls occurs simultaneously with the strain-driven migration of Inrich material towards protrusions at the NW ridges. Therefore nanoscale strain gradients are the major force driving the In segregation at the ridges of lattice-mismatched NW heterostructures.

We also observe that In is poorly incorporated at the highly Al-rich stripes. We attribute this to the low miscibility of In with Al-containing alloys [45]. At the $\{112\}$ nanofacets, In competes with $\mathrm{Al}$ for the available binding sites. This is particularly relevant for the B-polar nanofacets because $\mathrm{Al}$ tends to accumulate more in these positions. Throughout the shell, the In segregation strongly correlates with polarity: regions with a markedly higher In fraction coincide with the B-polar vertices. We note that the polarity-driven incorporation of In has not been demonstrated in the past. The competition between In and $\mathrm{Al}$ to stick on the $\{112\}$ nanofacets strongly favors $\mathrm{Al}$ on the B-polar surfaces: this mechanism may generate a net flux of In atoms towards the surroundings of the $\{112\} \mathrm{B}$ Al-rich planes. The literature on GaAs-AlGaAs core-shell NWs also reports that the $\{112\} \mathrm{B}$ nanofacets are systematically wider than the $\{112\}$ A ones $[18,30]$; concomitantly, the surface-curvature gradient from the $\{110\}$ to the $\{112\}$ facets, depends on the polarity of the $\{112\}$ nanofacet [30]. The polarity-dependent width and curvature of the NW surface may facilitate the In incorporation in proximity of the B-polar directions by directly acting of the local NW surface energy or by influencing the strain relaxation.

Conclusion. In conclusion, we have demonstrated a significant $(300 \mathrm{meV})$ redshift of the emission energy of singlephoton emitters embedded in the shell of core-shell GaAsAlGaInAs NWs. The redshifting mechanism is based on the In incorporation in the AlGaAs shell alloy to form a quaternary semiconductor of lower band gap. The spatial distribution of different species in the shell is determined by several highresolution techniques of compositional analysis. In addition to the well-known $\mathrm{Al}$ and $\mathrm{Ga}$ segregation, we find evidence of novel wedge-shaped In-rich segregation. We explain the distribution of the different species in the quaternary alloy with their diffusion on the NW sidewalls driven by crystal phase, polarity, and strain. Finite-element simulations provide insight in the role of strain to drive the segregation of In. These findings advance the understating of the segregation phenomena in quaternary alloys, as required to take full advantage of the additional degrees of freedom that they offer.

Acknowledgments. LF thanks the Nextnano team for the prompt support with the simulations; JSR thanks the European Synchrotron Radiation Facility (ESRF) for the beamtime allocated on the beamline ID16B. LF, GT, SES, HP, MF, and AFiM thank SNF for funding through NCCR QSIT, IZLRZ2 _163861 and BSCGI0_ 157705 and H2020 for funding through grant LIMQUET. EDR and LR thank Labex EMC3 AQURATE (French ANR). SMS acknowledges funding from "Programa Internacional de Becas 'la Caixa'-Severo Ochoa." JA and SMS acknowledge funding from Generalitat de Catalunya 2017 SGR 327 and the Spanish MINECO project ENE2017-85087-C3. ICN2 acknowledges support from the Severo Ochoa Programme (MINECO, Grant No. SEV-2013-0295) and is funded by the CERCA Programme / Generalitat de Catalunya. Part of the present work has been performed in the framework of Universitat Autònoma de Barcelona Materials Science PhD program. Part of the HAADF-STEM microscopy was conducted in the Laboratorio de Microscopias Avanzadas at Instituto de Nanociencia de Aragon-Universidad de Zaragoza. Authors acknowledge the LMA-INA for offering access to their instruments and expertise. This work has received funding from the European Union's Horizon 2020 Research and Innovation Programme under grant agreement No. 654360 NFFA-Europe. The authors declare no financial interest. 
[1] K. Onabe, Jpn J. Appl. Phys. 21, L323 (1982).

[2] G. B. Stringfellow, J. Cryst. Growth 65, 454 (1983).

[3] J. W. Matthews and A. E. Blakeslee, J. Cryst. Growth 27, 118 (1974).

[4] M. Leyer, J. Stellmach, C. Meissner, M. Pristovsek, and M. Kneissl, J. Cryst. Growth 310, 4913 (2008).

[5] R. B. Lewis, L. Nicolai, H. Küpers, M. Ramsteiner, A. Trampert, and L. Geelhaar, Nano Lett. 17, 136 (2017).

[6] O. Salehzadeh, K. L. Kavanagh, and S. P. Watkins, J. Appl. Phys. 114, 054301 (2013).

[7] S. Raychaudhuri and E. T. Yu, J. Vac. Sci. Technol. B 24, 2053 (2006).

[8] T. E. Trammell, X. Zhang, Y. Li, L. Q. Chen, and E. C. Dickey, J. Cryst. Growth 310, 3084 (2008).

[9] K. L. Kavanagh, Semicond. Sci. Technol. 25 (2010).

[10] M. De La Mata, C. Magén, P. Caroff, and J. Arbiol, Nano Lett. 14, 6614 (2014).

[11] F. Glas, Phys. Rev. B 74, 121302(R) (2006).

[12] F. Glas, in Lattice Engineering: Technologies and Applications, edited by S. M. Wang (Pan Stanford, Singapore, 2012), Chap. 5.

[13] T. Kuykendall, P. Ulrich, S. Aloni, and P. Yang, Nat. Mater. 6, 951 (2007).

[14] M. De La Mata, R. Leturcq, S. R. Plissard, C. Rolland, C. Magén, J. Arbiol, and P. Caroff, Nano Lett. 16, 825 (2016).

[15] M. Heiss, Y. Fontana, A. Gustafsson, G. Wüst, C. Magen, D. D. O'Regan, J. W. Luo, B. Ketterer, S. Conesa-Boj, A. V. Kuhlmann, J. Houel, E. Russo-Averchi, J. R. Morante, M. Cantoni, N. Marzari, J. Arbiol, A. Zunger, R. J. Warburton, and A. Fontcuberta i Morral, Nat. Mater. 12, 439 (2013).

[16] L. Mancini, Y. Fontana, S. Conesa-Boj, I. Blum, F. Vurpillot, L. Francaviglia, E. Russo-Averchi, M. Heiss, J. Arbiol, A. Fontcuberta I Morral, and L. Rigutti, Appl. Phys. Lett. 105, 243106 (2014).

[17] L. Francaviglia, Y. Fontana, and A. Fontcuberta i Morral, Quantum Dots in Nanowires, edited by S. A. Dayeh, A. Fontcuberta i Morral, and C. Jagadish, Semiconductors and Semimetals, Vol. 94 (Academic Press, Burlington, 2016), pp. 159-184.

[18] C. Zheng, J. Wong-Leung, Q. Gao, H. H. Tan, C. Jagadish, and J. Etheridge, Nano Lett. 13, 3742 (2013).

[19] N. Sköld, J. B. Wagner, G. Karlsson, T. Hernán, W. Seifert, M.-E. Pistol, and L. Samuelson, Nano Lett. 6, 2743 (2006).

[20] D. Rudolph, L. Schweickert, S. Morkötter, L. Hanschke, S. Hertenberger, M. Bichler, G. Koblmüller, G. Abstreiter, and J. J. Finley, New J. Phys. 15, 113032 (2013).

[21] E. H. Li, Physica E 5, 215 (2000).

[22] I. Vurgaftman, J. R. Meyer, and L. R. Ram-Mohan, J. Appl. Phys. 89, 5815 (2001).

[23] C. Colombo, D. Spirkoska, M. Frimmer, G. Abstreiter, and A. i Morral, Phys. Rev. B 77, 155326 (2008).

[24] T. V. Hakkarainen, A. Schramm, J. Mäkelä, P. Laukkanen, and M. Guina, Nanotechnology 26, 275301 (2015).
[25] M. Heigoldt, J. Arbiol, D. Spirkoska, J. M. Rebled, S. ConesaBoj, G. Abstreiter, F. Peiró, J. R. Morante, and A. i Morral, J. Mater. Chem. 19, 840 (2009).

[26] A. i Morral, D. Spirkoska, J. Arbiol, M. Heigoldt, J. R. Morante, and G. Abstreiter, Small 4, 899 (2008).

[27] L. Francaviglia, Y. Fontana, S. Conesa-Boj, G. Tütüncüoglu, L. Duchêne, M. B. Tanasescu, F. Matteini, and A. Fontcuberta i Morral, Appl. Phys. Lett. 107, 033106 (2015).

[28] L. Francaviglia, G. Tütüncüoglu, F. Matteini, and A. Fontcuberta i Morral, Nanotechnology 30, 054006 (2019).

[29] N. Jeon, B. Loitsch, S. Morkoetter, G. Abstreiter, J. Finley, H. J. Krenner, G. Koblmueller, and L. J. Lauhon, ACS Nano 9, 8335 (2015).

[30] N. Jeon, D. Ruhstorfer, M. Döblinger, S. Matich, B. Loitsch, G. Koblmueller, and L. J. Lauhon, Nano Lett. 18, 5179 (2018).

[31] Y. Fontana, P. Corfdir, B. Van Hattem, E. Russo-Averchi, M. Heiss, S. Sonderegger, C. Magen, J. Arbiol, R. T. Phillips, and A. Fontcuberta i Morral, Phys. Rev. B 90, 075307 (2014).

[32] See Supplemental Material at http://link.aps.org/supplemental/ 10.1103/PhysRevMaterials.3.023001 for further statistics on the NW composition as well as PL and Raman spectroscopy data.

[33] P. Trebbia and N. Bonnet, Ultramicroscopy 34, 165 (1990).

[34] I. T. Jolliffe, Principal Component Analysis, 2nd edn. (SpringerVerlag, New York, 2002).

[35] L. Balaghi, G. Bussone, R. Grifone, R. Hübner, J. Grenzer, and A. Krasheninnikov, arXiv:1803.10873.

[36] For the elements here considered, the EELS peaks overlap with an important background that is absent in the EDS spectra. The EELS background does not prevent the localization of the elements in the maps, but may reduce the accuracy of the quantification.

[37] F. Boxberg, N. Søndergaard, and H. Q. Xu, Adv. Mater. 24, 4692 (2012).

[38] A. Trellakis, T. Zibold, T. Andlauer, S. Birner, R. K. Smith, R. Morschl, and P. Vogl, J. Comput. Electron. 5, 285 (2006).

[39] S. Birner, T. Zibold, T. Andlauer, T. Kubis, M. Sabathil, A. Trellakis, and P. Vogl, IEEE Trans. Electron Devices 54, 2137 (2007).

[40] J. Grönqvist, N. Søndergaard, F. Boxberg, T. Guhr, S. Åberg, and H. Q. Xu, J. Appl. Phys. 106, 053508 (2009).

[41] P. Pfeffer, I. Gorczyca, and W. Zawadzki, Solid State Commun. 51, 179 (1984).

[42] S. Koshiba, Y. Nakamura, M. Tsuchiya, H. Noge, H. Kano, Y. Nagamune, T. Noda, and H. Sakaki, J. Appl. Phys. 76, 4138 (1994).

[43] Y. Kim, H. J. Joyce, Q. Gao, H. H. Tan, C. Jagadish, M. Paladugu, J. Zou, and A. A. Suvorova, Nano Lett. 6, 599 (2006).

[44] D. E. Jesson, S. J. Pennycook, J.-M. Baribeau, and D. C. Houghton, Phys. Rev. Lett. 68, 2062 (1992).

[45] P. Ballet, J. B. Smathers, and G. J. Salamo, Appl. Phys. Lett. 75, 337 (1999). 